Received: December21,2016 Accepted: January10,2017 Published: January23,2017

\section{Mechanism of action of the biologics used in the treat- ment of plaque psoriasis}

\author{
Ana Paula Galli Sanchez ${ }^{1 *}$, Camila Oliveira Alvarenga ${ }^{2}$ \\ ${ }^{1}$ Dermatologist and Master of Science from the Medical College of the University of São \\ Paulo; São Paulo-SP, Brazil \\ ${ }^{2}$ Medical doctor, Resident at the Dermatology Department in Hospital Ipiranga, São \\ Paulo-SP, Brazil
}

*Corresponding author: Ana Paula Galli Sanchez, Dermatologist and Master of Science from the Medical College of the University of São Paulo; Medical contributor to severe psoriasis clinic of the Hospital Ipiranga, São Paulo-SP, Brazil, Tel: 5511961753232; E-mail: apc-med@hotmail.com

\section{Abstract}

The biologics modified the treatment of plaque psoriasis in recent years. It is important that dermatologists know the mechanism of action of these drugs so they can better understand their indications and side effects. In this article we will discuss the mechanism of action of five biologics used in the treatment of plaque psoriasis: three anti-tumour necrosis factor agents (infliximab, adalimumab and etanercept) and two interleukin inhibitors (ustekinumab and secukinumab).

\section{Keywords:}

Biological Factors; Therapeutics; Psoriasis; Interleukin23; Interleukin-17; Tumor Necrosis Factor-alpha;

\section{Introduction}

Tumour necrosis factor-alpha (TNF- $\alpha$ ) is a potent proinflammatory cytokine exerting pleiotropic effects on various cell types and plays a critical role in the pathogenesis of chronic inflammatory diseases, such as psoriasis $[1,2]$. Accumulating evidence suggests that not only soluble TNF- $\alpha$ (sTNF, a homotrimer of $17 \mathrm{kDa}$ monomers), but also its precursor form (transmembrane TNF [tTNF], a homotrimer of $26 \mathrm{kDa}$ monomers) is involved in the inflammatory response $[1,2]$. sTNF is released as a soluble cytokine after being enzymatically cleaved from its cell-surface-bound form (tTNF) by TNF- $\alpha$-converting enzyme[1, $2]$.

TNF is produced by numerous cell types, including immune cells (B cells and T cells, basophils, eosinophils, dendritic cells, natural killer cells, neutrophils and mast cells), nonimmune cells (astrocytes, fibroblasts, glial cells, granuloma cells and ker- atinocytes) and many kinds of tumor cells [2]. The biological activity of TNF- $\alpha$ is triggered by binding to one of two structurally distinct receptors: TNF receptor type I (TNFRI [or p55 or CD120a]) and TNF receptor type II (TNFRII [or p75 or CD120b]) $[1,2]$. TNFRI and TNFRII are present in all cell types except erythrocytes $[1,2]$. Upon binding to TNF receptors, both transmembrane and soluble TNF- $\alpha$ mediate pleiotropic effects (apoptosis, cell proliferation and cytokine production) $[1,2]$.

Three anti-TNF agents, Infliximab (INF), Adalimumab (ADA) and Etanercept (ETN) are approved worldwide for the treatment of psoriasis. INF and ADA are anti-TNF monoclonal antibodies [1, 2]. INF is a human-murine chimeric monoclonal antibody with a constant human region $(\mathrm{Fc})$ and a variable mouse region and ADA is a fully human IgG1 monoclonal anti-TNF antibody [1, 2]. Both have two binding sites for TNF- $\alpha$ and present high specificity, affinity and avidity for the citokyne $[1,2]$. ETN is composed of the extracellular portion of two human TNFRII linked to a Fc portion (CH2 and CH3 domains) of human IgG1. ETN is supposed to form 1:1 complex with the TNF- $\alpha$ trimer [1]. INF and ADA form stable complexes with TNF- $\alpha$, while ETN forms relatively unstable complexes [1].

The TNF- $\alpha$-producing cells temporarily express TNF- $\alpha$ in their plasma membranes (tTNF) [1, 2]. INF, ADA and ETN bind to transmembrane TNF- $\alpha$ with similar affinities that are lower (weaker) than for soluble TNF- $\alpha$ [1]. Since INF and ADA are IgG1 antibodies, binding to tTNF, they are capable of complement fixation and also can produce the destruction of the TNF- $\alpha$-bearing cell by antibody dependent cell cytotoxicity (ADCC) $[1,2]$. ETN possess the Fc portion of IgG1 that can induce ADCC, but it does not carry the $\mathrm{CH} 1$ domain of IgG1 which is important for the activation of C3 [1]. Thus, differential clinical efficacies of anti-TNF agents may be explained by their different action on transmembrane TNF- $\alpha$-bearing cells [1].

The two main sources of TNF- $\alpha$ in the body are lymphocytes 
and macrophages (cells which form the granulomas) and the sTNF is essential for the maintenance of granulomas architecture [1-3]. ADA and INF induce complement-dependent cytotoxicity, ADCC and outside-to-inside signalling through transmembrane TNF- $\alpha$ and seem to be more potent than ETN in the elimination of transmembrane TNF- $\alpha$-bearing macrophages and transmembrane TNF- $\alpha$-bearing T cells. Therefore, they are very effective in disrupting granulomas, being indicated in the treatment of granulomatous diseases such as Crohn's disease [1]. However, the occurrence of reactivation of latent TB is more common in patients receiving ADA and INF than in patients treated with ETN or with other biologics that do not directly inhibit the TNF- $\alpha$ (for example, the interleukin inhibitors) [3].

ADA and INF are more effective than the ETN in the treatment of plaque psoriasis [4]. The most common adverse effects of ADA and ETN (subcutaneous use) are reactions in application sites while the most common adverse effects related to INF (intravenous use) are infusion reactions [5]. Another common side effect of anti-TNF agents are common infections (especially of the upper respiratory tract) [5]. As a result of the immunological alterations provoked by TNF- $\alpha$ inhibitors, the use of these drugs has been associated with severe infections of viral, bacterial and fungal etiology so it is essential to properly monitor the patients using these drugs and to know all their common and rare possible adverse effects [1-3, 5].

The inhibitors of interleukins (ustekinumab - UST and secukinumab - SEC) are more effective in the treatment of plaque psoriasis than the anti-TNF agents $[4,6]$ as they act directly on the Interleukin (IL) -23/17 axis (the protagonist of the immunopathogenesis of psoriasis) [7]. By the way, the main mechanism of action of the anti-TNF agents in plaque psoriasis appears to be the inhibition of sTNF involved in the activation of dermal dendritic cells (which are potent sources of IL-23). Thus, the anti-TNF agents would also inhibit the IL-23/17 axis [7].

Ustekinumab is a fully human IgG1-k monoclonal antibody that binds specifically to the p40 subunit of the cytokines interleukin (IL)-12 (p40+p35) and IL-23 (p40+p19). It binds to the same epitope within the D1 domain of the p40 subunit of each cytokine. Binding of ustekinumab to IL-12 and IL-23 prevents their association with IL- $12 \mathrm{R} \beta 1$, which is expressed on the surface of a variety of immune cells such as natural killer and T cells. By directly neutralizing their biological activity, ustekinumab attenuates the immune cell activation properties of IL-12 and IL-23. Ustekinumab is unable to bind to IL-12 or IL-23 that is already bound to IL$12 \mathrm{R} \beta 1$; therefore, it is unlikely to contribute to complement- or antibody-mediated cytotoxicity [8]. By inhibiting the soluble IL12 and IL-23, UST inhibits, respectively, the differentiation in the lymph node of naive T-helper lymphocytes (LTh $\theta$ ) into Th1 and Th17 [7-9]. In psoriasis patients, these activated Th17 and Th1 cells fall in circulation and are captured by the activated endothelial cells on the skin. In the dermis, Th17 and Th1 interact with the antigen-presenting cells - APC (i.e, macrophage and dermal dendritic cells) and under the influence of IL-12 and IL-23 (produced by the APC), they proliferate and release their specific repertoire of cytokines (TNF- $\alpha$, INF $\gamma$, IL-17, IL-22 and others) [7, 9]. By inhibiting IL-23 and IL-12, UST does not allow the prolif- eration of the Th1 and Th17 in the dermis. Besides, UST may also act in skin lesions preventing IL-17 release by various cells of the innate immune system (neutrophils, mast cells, LTY $\delta$ cells and innate lymphoid cells) [7, 10]. Finally, it is a very effective drug [4] and has an excellent safety profile for the treatment of plaque psoriasis. Nasopharyngitis, upper respiratory tract infection and headache are reported as the most common adverse effect of UST $[5,9,11]$.

IL-17 (or IL-17A) is a key cytokine in the immunopathogenesis of psoriasis [7]. It acts on keratinocytes altering their differentiation and proliferation, and stimulating them to produce various proteins (cytokines, chemokines and antimicrobial peptides), which attracts more immune cells to the skin [7]. IL-17A acts on keratinocytes individually and together with the TNF- $\alpha$ and IL-22 [7].

The inhibition of IL-17 by SEC (human IgG1 $\kappa$ monoclonal antibody that binds to soluble interleukin IL-17A) reduces the production of various chemokines by keratinocytes including the ones responsible for the arrival of neutrophils in the skin $[6,12]$. SEC causes rapid disappearance of neutrophils (potent sources of IL17A) in psoriasis lesions [12]. The disappearance of neutrophils correlates with the decrease in proliferation of keratinocytes, demonstrating a strong interaction between these cells in the immune response [12]. IL-17A is important in defense against extracellular pathogens and candidiasis has been reported in patients using SEC (cases controlled with classical treatments without systemic infection report) $[6,12]$. The effectiveness of SEC is greater than the UST in the treatment of plaque psoriasis [6], but rare adverse effects of SEC include neutropenia and isolated reports of Crohn's disease activation $[6,12]$.

\section{Conclusion}

In conclusion, it is important to know the immunopathogenesis of psoriasis and the mechanisms of action of the biologics in order to understand better their indications and possible adverse effects. In this way, we can individualize the treatment of those patients who need this type of medication.

\section{References}

1. Horiuchi T, Mitoma H, Harashima S, Tsukamoto H, Shimoda T. Transmembrane TNF- $\alpha$ : structure, function and interaction with anti-TNF agents. Rheumatology. 2010;49(7):1215âĂŞ1228. doi: 10.1093/rheumatology/keq031

2. Silva LCR, Ortigosa LCM, Bernard G. Anti-TNF-a agents in the treatment of immune-mediated inflammatory diseases: mechanisms of action and pitfalls. Immunotherapy. 2010;2(6):817-833. doi: 10.2217/imt.10.67.

3. Cantini F, Nannini C, Niccoli L, Iannone F, Delogu G, Garlaschi $\mathrm{G}$, et al. Guidance for the management of patients with latent tuberculosis infection requiring biologic therapy in rheumatology and dermatology clinical practice. Autoimmunity Reviews. 2015;14(6):503-509. doi: 10.1016/j.autrev.2015.01.011 
4. Puig L, Lopez A, Vilarrasa E, Garcia I. Efficacy of biologics in the treatment of moderate-to-severe plaque psoriasis: a systematic review and meta-analysis of randomized controlled trials with different time points. J Eur Acad Dermatol Venereol. 2014;28(12):1633-1653. doi: 10.1111/jdv.12238

5. Sehgal VN, Pandhi D, Khurana A. Biologics in dermatology: adverse effects. Int J Dermatol. 2015;54(12):1442-1460. doi: 10.1111/ijd.12802

6. Blauvelt A, Reich K, Tsai TF, Tyring S, Vanaclocha F, Kingo $\mathrm{K}$, et al. Secukinumab is superior to ustekinumab in clearing skin of subjects with moderate-to-severe plaque psoriasis up to 1 year: Results from the CLEAR study. Journal of the American Academy of Dermatology. 2017;76(1):60-69. doi: 10.1016/j.jaad.2016.08.008

7. Yaxiong Deng Y, Chang C, Lu Q. The inflammatory response in psoriasis: a comprehensive review. Clinic Rev Allerg Immunol. 2016;50(3):377-389. doi: 10.1007/s12016-016-8535-x.

8. Croxtall JD. Spotlight on ustekinumab in moderate to severe plaque psoriasis. Am J Clin Dermatol. 2012;13(2):135-137. doi: 10.2165/11208650-000000000-00000.
9. Benson JM, Sachs CW, Treacy G, Zhou H, Pendley CE, Brodmerkel CM, et al. Therapeutic targeting of the IL-12/23 pathways: generation and characterization of ustekinumab. Nat Biotechnol. 2011;29(7):615-624. doi: 10.1038/nbt.1903.

10. Lin AM, Rubin CJ, Khandpur R, Wang JY, Riblett M. Mast cells and neutrophils release IL-17 through extracellular trap formation in psoriasis. J Immunol. 2011;187(1):490-500. doi: 10.4049/jimmunol.1100123

11. Alice BG, Robert EK, Richard GL, Gerald GK, Elke MGJJ, LynnG et al. Safety Observations in 12095 Patients With Psoriasis Enrolled in an International Registry (PSOLAR): Experience With Infliximab and Other Systemic and Biologic Therapies. J Drugs Dermatol. 2014;13(12):1441-1448.

12. Kristian Reich K, Papp KP, Matheson RT, Tu JH, Bissonnette R, Bourcier M, et al. Evidence that a neutrophilâĂŞkeratinocyte crosstalk is an early target of IL-17A inhibition in psoriasis. Experimental Dermatology. 2015;24(7):529-535. doi: 10.1111/exd.12710. 